\title{
AUTISMO INFANTIL: EL ESTADO DE LA CUESTIÓN
}

\section{Maricruz Coto Choto*}

\section{RESUMEN}

Este artículo constituye una revisión bibliográfica de investigaciones realizadas sobre el autismo infantil. Desde una perspectiva psicológica, se describe el estado de la cuestión en el tema respecto a: dificultades en la conceptualización y las consecuencias en el diagnóstico; metodologías y procedimientos a los que se ha recurrido en la investigación y los temas que han sido ejes centrales de esta.

\section{PALABRAS CLAVE: AUTISMO $*$ NIÑEZ * DIAGNÓSTICO DIFERENCIAL * INVESTIGACIÓN}

\begin{abstract}
This article constitutes a bibliographical revision of investigations done about child autism. It describes from a psychological perspective, the state of the investigations, with regards to difficulties in the conceptualization and the consequences in the diagnose, methodologies and procedures that have been recurred to in the investigation and topics that have been turning points for the investigation.
\end{abstract}

KEY WORDS: AUTISM $*$ CHILDREN $*$ DIFFERENTIAL DIAGNOSIS * INVESTIGATION

\section{INTRODUCCIÓN}

El panorama de la investigación en el tema del autismo infantil es confuso y amplio lo cual evidencia la complejidad del trastorno. La causa, el pronóstico y el tratamiento son todavía tema de estudio en las comunidades científicas.
En este trabajo, se pretende dar cuenta del estado de la investigación en el autismo infantil, en cuanto a las dificultades en su definición y diagnóstico; se realiza además una exploración de las diversas técnicas e instrumentos utilizados en su tratamiento, así como las metodologías y procedimientos más empleados en la investigación; $y$, 
en cada uno de los ejes anteriores, se evidencian las temáticas abordadas con mayor frecuencia.

Actualmente se identifica al síndrome autista de varias maneras: sindrome de Kanner, autismo infantil precoz, autismo anormal primario, autismo encapsulado secundario, esquizofrenia de tipo autista, desarrollo atípico de la niñez con rasgos autistas y retraso mental asociado con autismo. Debido al desconocimiento de su origen, el autismo se halla rodeado de una gran controversia con respecto a su diagnóstico y a su probable etiología. Como es una condición rara, los primeros estudios se basaron en pocos casos, $y$ hasta hace pocos años, se le ha ido dedicando mayor atención.

\section{ANTECEDENTES}

El término autismo, proviene del griego autos que significa sí mismo, y refiere a la expresión de "ausente o perdido". Fue utilizado por primera vez por Bleuler en 1911 (1985) para referirse a un trastorno del pensamiento que aparece en algunos pacientes esquizofrénicos $y$ que consiste en la continua autorreferencia que hacen de estos sujetos a cualquier suceso que ocurre. Sin embargo, este síntoma, tal y como lo acuñó Bleuler, no es posible aplicarlo al autismo infantil porque describía a adultos que tenían regresiones a un nivel psíquico inferior, por lo que un niño en el inicio de su vida no vive las regresiones descritas por este autor. Por este motivo, aunque otros autores tiempo atrás habían observado conductas autistas en niños, se considera a Kanner (1957) el pionero en la literatura existente sobre este trastorno infantil. Kanner realizó observaciones en niños que sufrían alteraciones extrañas, las cuales no estaban descritas en ningún sistema nosológico; estas conductas eran coincidentes entre sí $y$ diferentes del resto de los niños con alteraciones psicopatológicas.

Kanner considera el autismo como un síndrome comportamental que se manifiesta por una alteración del lenguaje, de las relaciones sociales y los procesos cognitivos en las primeras etapas de la vida. Para este autor la sintomatología radica en la alteración del contacto socioafectivo, lo que supuso que en las dos décadas posteriores la mayor parte de las investigaciones identificaran al autismo con trastornos emocionales, enfoque que ha desarrollado profusamente la escuela psicoanalítica. A partir de los años sesenta comienzan a diversificarse las líneas de investigación, conduciendo a una visión más compleja del autismo.

La investigación de Kanner es fundamental, ya que hasta su descripción el síndrome adquiere el nombramiento como categoría diagnóstica en 1943. Desde entonces el concepto ha sufrido innumerables modificaciones según el abordaje que se le otorga y todavía en la actualidad este punto resulta controversial.

\section{LA PROBLEMÁTICA DE DEFINIR EL AUTISMO Y SUS CONSECUENCIAS EN EL DIAGNÓSTICO}

La definición del "autismo" varía según el enfoque, por lo cual es fácil encontrar diferencias en cuanto a las características otorgadas al trastorno, según sea el área de interés; por ejemplo desde un abordaje educativo existe la idea que establece que estos cuadros son acompañados siempre de algún grado de retraso mental, lo cual ha condicionado un abordaje terapéutico que privilegia los programas educacionales. Una definición de tipo comportamental como la de Creak (1960) o la de Rutter y Schopler (1984) ofrece criterios útiles para planificar un programa individual, ya que las variaciones en los tipos y grados de anomalías son enormes. Así, cuando el objetivo de la clasificación de un niño es encontrar para él un aula o un programa educacional adecuados, vemos que algunas conductas "autistas" son más significativas que otras. En 1964 Creak (citado por Paluszny, 1987), en un intento por afinar criterios y evitar errores de diagnóstico, propuso una "lista de síntomas" que sirviera de orientación y fijara criterios diagnósticos, con base en 9 puntos. Pero esta enumeración, que no era gradativa, sino que todos los items eran considerados de igual importancia, fracasó por ser de una excesiva generalidad y de escasa especificidad. Otros autores han realizado similares intentos sin mucho éxito (Polan y Spencer (1959), Lotter (1966), De Myer y 
Churchill (1971), citados por Paluszny, 1987). Estos esfuerzos fueron comparados y se llegó a la conclusión de que niños que puntuaban altas conductas autistas en una escala, recibían una puntuación baja en otra, por lo que se superponían áreas de clasificación. Los criterios de Rutter (1984) con algunas modificaciones, confirman los de Kanner, (1957) y son los más utilizados en el diagnóstico.

La posición de Rutter (1984) puede resumirse de la siguiente forma: atribuye a los trastornos del lenguaje el carácter de alteración primaria, partiendo de un daño orgánico cerebral. Afirma también que otros trastornos de la percepción son probablemente importantes, subrayando en cambio, que la psicosis no es primariamente emocional en su origen, $y$ afirmando además que los factores psicógenos sólo desempeñan un papel secundario. Un aporte importante es que diferencia la psicosis infantil de la esquizofrenia del adulto, aspecto que representa una de las principales confusiones en las que se ha incurrido; la duda básica es respecto a si este síndrome es la temprana manifestación de la esquizofrenia. Existen tendencias que consideran que diferentes psicodinamias permiten darle ubicaciones diversas (Delgado, 1996). Klein, (1948) insiste en la diferenciación entre las psicosis del adulto $y$ las infantiles, ya que la relación de uno y otro con la realidad es radicalmente distinta.

Desde la psicología, las principales definiciones que se toman son las dadas por Rutter en 1984 (citado por Rodríguez, 1992), quien refiere un conjunto de características y criterios a tomar en cuenta a la hora de definir el autismo, tales como: comienzo antes de los treinta meses de edad, desarrollo social alterado, desarrollo lingüístico retrasado y anómalo, pautas de juego estereotipadas, $y$ resistencia al cambio. Otros autores como Eisenbert, Kanner y Schopler (citados por Calderón y Chacón, 2000), Paluszny y Fonseca (citados por Chacón, $y$ otros, 1994) coinciden con esta definición básica, otorgando mayor o menor importancia a algunos elementos. En general las definiciones son congruentes al destacar un problema básico en el desarrollo.

Las tendencias se han desarrollado a lo largo de diversos países y según las distintas corrientes. En lo que respecta a Estados Unidos, se pueden encontrar dos posiciones opuestas claramente definidas, que han dirigido la investigación; representadas por L. Bender (1965), biologista quien argumenta una desorganización en el sistema nervioso central y considera el autismo como un retraso en la maduración y como esquizofrenia infantil; y M. Mahler (1975), de la escuela psicoanalítica, quien destaca la relación simbiótica madre-hijo en la génesis de la psicosis, por lo que el autismo deviene de la ruptura en esta relación. Tal como señala Tustin (1972), los niños viven una situación catastrófica cuando son conscientes de su separación con la madre, ante lo cual desarrollan el "cascarón protector". Este autor propone, en 1991, dos tipos de autismo: en el primero, predominan los factores orgánicos, mientras que en el segundo los factores psicógenos $y$, además distingue tres formas: 1) primario anormal, 2) secundario encapsulado, y 3) secundario regresivo, las cuales han sido utilizadas por sus seguidores como parámetros de diagnóstico y pronóstico (Trejo, 1996).

En general la escuela psicoanalítica se inclina a considerar el autismo como un producto de una defectuosa comunicación y falta de entendimiento entre los adultos y el bebé en sus primeros momentos de vida. Asimismo desde esta postura se plantean distintas concepciones en cuanto a las causas y a la estructura en juego. El psicoanálisis muestra algunas limitaciones e insuficiencias para la atención de los niños autistas, pero ha ido desarrollando junto a un mayor conocimiento de estas enfermedades, nuevas modalidades técnicas para su tratamiento. Algunas consideraciones importantes las aporta Bettelheim (1977), psicoanalista austriaco, para quien los niños que sufren autismo se encuentran detenidos en un período inicial de su desarrollo intelectual y emocional; considerando el autismo como una reacción ante un extremo aislamiento afectivo combinado con experiencias interpretadas como amenazadoras para su existencia. Uno de los conceptos centrales de su teoría es el concepto de sí mismo, que explica como los niños autistas se han retirado del mundo para volverse hacia sí mismos, de aquí, la tarea del terapeuta es ayudarlos a invertir el proceso psicótico creando un mundo 
diferente del abandonado a causa de la desesperación, lo cual implica una escucha constante, un acompañamiento del niño en sus fantasmas $y$ el respeto de sus síntomas y comportamientos. Este autor ofrece una perspectiva interesante para comprender el "interior" de los niños autistas y la desesperación de que son víctima, esta angustia les hace buscar seguridad evitando el contacto, $y$ pueden inclusive no reconocer su hostilidad, por esta razón sus defensas hacia el mundo externo amenazador fallan, $y$ lo único que los protege es el no actuar; el niño se insensibiliza tanto hacia el exterior como hacia su psiquismo. De aquí la importancia de la relación madre-hijo. Bettelheim (ibid) describe al autismo como un trastorno de la comunicación, donde el niño interpreta mal las señales que el mundo y la madre le envían, $y$ esta persistencia del fracaso en enviar y recibir mensajes son los determinantes de la gravedad de la perturbación. Esto genera para el niño una angustia agobiante, va rompiendo lazos con la realidad exterior y la posición autística es el supremo esfuerzo por salvar la existencia, pero destruye el sí mismo. Debido a esta teoría, al autor se le ha culpado de promover la idea de la maternidad como responsable del autismo; sin embargo, otros autores atribuyen estas críticas al desconocimiento de su obra, donde uno de sus aportes fue precisamente reconocer la importancia de los factores paternos (Delgado, 1996).

Como reacción ante esta orientación se pueden citar las investigaciones acerca del apego, principalmente la de Capps, Sigman y Mundy en 1994 (Sánchez, 1996), quienes señalan que una muestra representativa de niños entre los 3 y los 6 años desarrollaron lo que ellos llaman "apego seguro", no aparente a primera vista, lo cual señala la existencia de una madre responsiva y afectuosa en algún momento. Por tanto, las conductas de apego aunque se manifiesten de forma desorganizada, son símbolo de un vínculo emocional. Respecto a este último aspecto es importante destacar la posición de Frith (1993, 59), quien dice que "los autistas no están fuera del alcance de las relaciones afectivas" lo cual viene a desmitificar la idea de que estos niños son seres aislados de la realidad $y$ del contacto con otras personas, aunque parezca que huyen del contacto afectivo con otros, sí lo viven y experimentan pues son capaces de sentir y vincularse, solo que de una manera especial, ya que su expresión se les dificulta y aún a su modo se relacionan con aquellos que le son significativos. Esta autora argumenta que hay abundantes ejemplos de niños que han recibido rechazo y deprivación sin que ninguno derive en autismo. Se concluye entonces que no se puede considerar el daño del vínculo materno como la única causa del autismo, sino que puede tener un peso importante en convergencia con otros factores. Pérez de Plá (1996) de la Asociación Mexicana para el Estudio del Retardo Mental y Psicosis Infantil, argumenta que estas críticas "probablemente representan intentos de negar aspectos dolorosos de la teoría tales como el lugar de la familia en la instalación del padecimiento" (ibid, 32). Para ella el campo autista se caracteriza por la "tendencia al desierto psíquico, desierto del aislamiento precoz de todo vínculo que impide el proceso de humanización" (ibid, 30), caracterizando a los autistas como incapaces de vincularse.

A pesar de las variantes, el punto central de los autores psicoanalíticos es la incidencia de los factores psicológicos y sociales, donde destaca con especial importancia, el vínculo materno que tiene la función de contención del pequeño $y$ es el que guía los primeros pasos hacia otros vínculos $y$ al desarrollo de las capacidades mentales y es, en el diagnóstico del autismo, un signo patognomónico innegable: la incapacidad para establecer un vínculo afectivo como lo hacen los otros niños.

Otros autores como Wing (1981), han dado la denominación de "espectro autista" la cual se ha considerado útil porque ubica al autismo en un amplio rango, o sea, se puede presentar en diversos grados y en diferentes trastornos como: la esclerosis tuberosa, enfermedades metabólicas, alteraciones genéticas como el síndrome X-frágil, etc. La importancia de este término radica en que permite tomar en cuenta la sintomatología autista en enfermedades muy diversas, precisar aproximadamente el grado, ligero, moderado o severo, y trazar pautas educativas. El equipo de investigadores de esta autora, ha realizado estudios demográficos en Londres, $y$ han señalado que 
los síntomas del autismo no se agrupan aleatoriamente, sino que hay una tríada específica de rasgos que se encuentran deteriorados: la comunicación, imaginación y socialización. Frith (1993) explica que esta tríada de alteraciones se explica por el fracaso de un único mecanismo, y añade este nuevo elemento descubierto por su grupo de investigaciones médicas: un componente cognitivo que se encuentra alterado en los niños autistas y es la capacidad de pensar o de imaginar el estado mental de otro individuo, lo cual se demuestra cuando los niños empiezan a participar en la denominada "atención compartida", que un niño autista es incapaz de mostrar. La alteración en este componente tiene graves consecuencias pues conlleva el impedimento de poder reflejarse a sí mismo.

En esta misma línea está Pérez de Plá, (1996) coincide al decir que el autismo es un síndrome que reúne diferentes cuadros patológicos. El peligro que se corre sigue siendo el mismo: las dificultades para el diagnóstico diferencial siguen estando presentes $y$ un error en ello llevaría implícito un tratamiento equivocado, con toda la repercusión que tendría para el niño, la familia y el resto del entorno.

El autismo se ha intentado definir también desde diversas institucionalidades como son la Sociedad Americana de Autismo (ASA), antes llamada National Society for Autistic Children (NSAC, 1977) que adopta una postura conductual: el autismo es un trastorno irreversible que inhabilita el normal desarrollo intelectual y emocional de la persona y que generalmente se manifiesta en los primeros tres años de vida (Fonseca, 1987). La irreversibilidad de este trastorno ha sido cuestionada por Sherzer (citado por Hernández, 1997) psicólogo de la Universidad de Montevideo en Uruguay, quien destaca que la principal causa del autismo es emocional y por esto puede ser reversible, lo que sí definitivamente les hace daño es la marginación de que son víctima.

Por otro lado el DSM IV lo considera como un desorden profundo en el desarrollo $y$ destacan entre sus características, el inicio en la niñez, la incapacidad para iniciar o aceptar contacto físico, evitación del contacto visual, impedimento cualitativo en la interacción social, en la comunicación verbal y no verbal y en la actividad imaginaria, y también restricción en el repertorio de actividades e intereses. La Organización Mundial de la Salud, brinda un enfoque útil, desde una perspectiva multiaxial, otorgando una clasificación combinada descriptiva-funcional, que incluye cuatro ejes: síndrome clínico, nivel intelectual, cualquier asociación o factores etiológicos-biológicos, y por último factores etiológicos-psicosociales (Paluszny, 1987).

Otros autores como Bernardo y Martín (1993, citado por Fernández y otros, 1999) proporcionan una definición bastante completa, caracterizándolo por ser un trastorno generalizado que aparece antes de los 30 meses de vida con alteraciones y déficit en la capacidad para relacionarse con las personas, en la utilización del lenguaje como comunicación social y la aparición de modelos de conductas repetitivas $y$ estereotipadas. A pesar de que actualmente no existe consenso entre las causas y conductas características y exclusivas del autismo; entre las muchas descripciones propuestas, $y$, a pesar de las discrepancias se puede observar un acuerdo al menos al señalar la presencia de alteraciones en la comunicación, el lenguaje y las relaciones sociales. Conductas "clásicas" que se mantienen en la mayoría de definiciones, cambiando la perspectiva según el énfasis que se le dé a uno u otro elemento.

Otras características se le han atribuido al síndrome, (Negrón, 1981): intolerancia al cambio de rutina, movimientos estereotipados, respuesta perceptual anormal, hipersensibilidad e hiposensibilidad ante determinados sonidos, menor uso de los sentidos de la vista y el oído y mayor uso del olfato, gusto y tacto. En estos aspectos se encuentran coincidencias con otros autores como Diez Cuervo y Martos (1989), Hobson (1993), (citados por Fernández y otros, 1999), Wing (1981), y Baron-Cohen y otros (1993).

Los estudios realizados demuestran que la variedad y la severidad de características cambian de un niño a otro, por lo que la heterogeneidad en las conductas complican la definición del autismo, el cual puede confundirse con otros síndromes como retardo, afasias, y 
psicosis, e incluso se han intentado establecer diferencias claras y específicas entre el síndrome de Asperger y el Autismo con el que posee grandes similitudes.

El diagnóstico del autismo se dificulta por varias razones: la publicidad de que ha sido objeto, la diversidad de aplicaciones que han recibido los criterios de diagnóstico, es decir la complejidad de sus características y la dificultad para identificarlas $y$ distinguirlas de otros síndromes; la falta de signos patognomónicos y la ausencia de indicadores psicobiológicos que puedan tomarse en cuenta para afirmar o negar el diagnóstico. La cuestión se complica pues la sintomatología del autismo suele encontrarse también en otras enfermedades $y$ por lo general presenta diferentes grados en las distintas etapas del desarrollo. Además de definiciones varias y poco precisas, está la heterogeneidad de la población y la gran variedad de instrumentos utilizados para el diagnóstico, lo que para algunos autores como Fernández y otros, (1999) resulta inadecuado.

En este punto es importante que se analice la prevalencia de esta patología, la cual se considera confusa dada la inexactitud de los diagnósticos. Existen estudios relativos a la epidemiología como los de Kanner en 1954, Creak e Ini (1960), Lotter en 1966 y Rutter en 1966 (citados por Lien de Rozental, 1983), los cuales indican que para los años noventas la incidencia de este síndrome era de 15 y 20 niños por cada 10000 nacimientos, y es cuatro veces más común en niños que en niñas, en las cuales siempre es necesario establecer el diagnóstico diferencial con el síndrome de Rett. Otros autores como Delgado (1996) cifran de 2-4 por cada 10000 entre las edades de los 0-12 ó 15 años; $y$ Baron-Cohen (1999) ofrecen datos aproximados de 4 a 15 niños por cada 10000 (ibid).

Poder diferenciar al autismo de otras condiciones, puede resultar una tarea muy ardua, puesto que existen problemas en varias áreas. Es muy complejo poder dilucidar, si el autismo es una entidad clínica específica, o tal vez una forma de esquizofrenia o de retraso mental. Además, puede ser complicado evaluar si el paciente es autista o padece otro trastorno. Kanner trabajó en el diagnóstico diferencial, reconociendo que la discrepancia básica entre el autista y el esquizofrénico, radica en que el segundo, en efecto, se retira del mundo, mientras que el primero, nunca logra entrar en él (citado por Bettelheim, 1977). Un reciente aporte lo brinda Rivière (citado por Guzmán, 1995), quien junto con un equipo de investigadores del Dpt. de Psicología de la Universidad Autónoma de Madrid, han trabajado en un "sistema experto" que sirve para diagnosticar casos de autismo utilizando los criterios dados por Kanner; la confiabilidad de este es de un $97 \%$, es decir que entre el diagnóstico realizado por el experto y el realizado por el sistema, sólo en tres casos diagnosticados, se está en desacuerdo. Este tipo de herramientas ayudan a solucionar el problema de conceptualización que presenta el síndrome.

Freeman (1981 citado por Rodríguez, 1992) establece tres tipos diferentes de estudios para realizar el diagnóstico: descripción clínica del síndrome, listas de conducta, escalas de clasificación y observación de la conducta en diferentes ambientes estructurados. Esta autora, junto con Ritvo (1984, ibid) establecen un procedimiento de diagnóstico diferencial que evalúa factores orgánicos, cognoscitivos-intelectuales, psicológicos y familiares-sociales.

Respecto a la etiología actualmente se han descartado varias teorías psicogenéticas como la de Kanner (1943, citado por Rodríguez, 1992) el cual le daba un peso trascendental al ambiente y relacionaba el comportamiento autista con la actitud fría y hostil de los padres (posteriormente esta posición fue modificada por él mismo en 1957, Kanner, 1957). Siguiendo esta misma orientación, Bettelheim (1977) y Tustin (1972) explican el autismo desde esta perspectiva, como una forma desviada reactiva de desarrollo. Estas teorías en su expresión más radical, aunque no dejan de tener sus seguidores, han sido eliminadas por una gran mayoría debido a lo escaso y contradictorio de sus pruebas.

Estudios como los de Schopler en 1973 (Rutter y Scholpler, 1984), ofrecen datos que contradicen la base de estas teorías. Este autor lleva a cabo una investigación que muestra como las personalidades de los padres de niños autistas tienen un rango normal de características de la personalidad. En este sentido, Paluszny (1987) afirma que no se han encontrado pruebas para ligar un tipo de personalidad 
peculiar de los padres, al comportamiento del niño, incluso no se han podido determinar ambientes específicos o modalidades particulares de interacción. Existen también estudios respecto a los patrones de crianza como el de DeMyer y Cols. 1972 (citados por Paluszny, 1987), que ponen al descubierto la falta de evidencias. Esta autora cita otro estudio importante, el de Ward en 1976 (ibid) quien sugiere una etiología de origen psicológico aunado a una deficiencia neurológica, donde la angustia de la madre durante el embarazo hace que el niño sea más lento en su desarrollo sensorial; esta teoría no está probada, pero sí se toma en cuenta las condiciones del parto como factor muy importante en el desarrollo del trastorno. Una posición radical la encontramos en Rimland (citado por Pérez de Plá, 1996), al hacer una fuerte crítica dirigida especialmente a los psicoanalistas, e insiste en mostrar que el autismo debe ser únicamente considerado desde su etiología biológica. Con lo cual no deja de situarse en un extremo que, si bien da importantes descubrimientos en la explicación del síndrome, no es suficiente para su entendimiento.

Otros autores buscan dar cuenta de correlatos fisiológicos, anatómicos, cognitivos y bio-químicos asociados al comportamiento autista. Al respecto hay teorías que intentan encontrar indicadores de un posible origen biogénico. Trabajos como los de Chess (1971 citado por Paluszny, 1987), consideran, por ejemplo, la rubéola durante el embarazo como causa de alta incidencia, la cual se da en aproximadamente un 5\% de los casos. En esta línea sobresalen los trabajos de Frith (1993), quien postula la existencia de una alteración biológica en virtud de la cual la mente del sujeto autista difiere notablemente de la de una persona normal. Otros estudios de autores como Freeman y Ritvo, 1984 (citado por Rodríguez, 1992), han encontrado datos sobre la posible influencia de factores genéticos como anomalías cromosómicas, e incluso enfermedades virales e infecciones intrauterinas que se han señalado como posibles causas del trastorno. También se habla de alteraciones al nivel de neurotransmisores $y$ de altos niveles de serotonina en la sangre (Ibid, 1978 y 1984) o estudios como el de Sankar en 1969 (citada por Lien de Rozental, 1983), quien demuestra una extensa ruptura de cromosomas en los leucocitos de niños autistas.

En cuanto a estas teorías no se busca que una sola sea la causa, sino que es factible pensar que al estar asociado con otros trastornos, las causas sean múltiples. Para Rivière (citado por Guzmán, 1995), es claro que en los últimos años ha quedado demostrado que el autismo es un trastorno biológico, $y$ aunque no se sabe a ciencia cierta la patogenia, ni las causas biológicas que pueden desarrollar el síntoma, las implicaciones biológicas son innegables. Por el momento se destacan tres áreas de gran interés: la genética, la bioquímica y la viral; donde resaltan pioneros del trabajo en estas áreas: Rimland, quien propone una teoría neurológica del autismo (1964), Goodwin (1969), Desmond (1970), Chess (1971) y Colman (1976) (citados por Paluszny, 1987).

Los estudios neurofisiológicos se han realizado principalmente en cinco áreas: estudios encefalográficos EEG, estudios de la respuesta sensorial evocada no contingente, estudios de la respuesta sensorial evocada que incluye contingencia, respuestas autonómicas y respuestas vestibulares. Todas estas investigaciones sugieren un fuerte componente motor en los procesos sensoriales de los niños autistas, lo cual señala una disfunción neurofisiológica que modula lo sensorial y lo motriz. Recientes estudios de Rivière (citado por Guzmán, 1995), demuestran la importancia del lóbulo frontal, el cual se encuentra alterado en su funcionamiento en las personas autistas; los últimos descubrimientos señalan que este lóbulo posee un período considerado como crítico para el desarrollo de sus capacidades y su funcionamiento, el cual coincide con el período crítico en el desarrollo del autismo, de ahí que estos niños sean incapaces de realizar tareas asociadas a su actividad.

Otros autores se han preocupado por realizar estudios en el área de la hematología como Lucas (1971), DeMyer (1968), Cohen (1976) (citados por Rutter, 1984) de lo cual los autores sugieren la necesidad de que se realicen réplicas de los estudios evaluando variables genéticas, culturales, sociales, dieta, e historia clínica. Como resultado de estos estudios se han elaborado escalas de puntuación y se descubrió el papel 
importante de la serotonina, cuyo metabolismo parece estar alterado en los niños autistas. Según los estudios revisados, la información disponible resulta insuficiente hasta el momento para determinar el cuadro autista debido a anomalías metabólicas. Por esta razón se destaca también la necesidad de realizar estudios longitudinales, que permitan establecer datos comparativos.

Comparando las posiciones anteriormente citadas, aunque se ha logrado superar la teoría de la relación madre-hijo como causa del autismo, y se ha podido entender los trastornos del desarrollo y especialmente del autismo infantil como no determinados por distorsiones en la conducta de los padres sino del sistema nervioso central, se contrapone el hecho de que a pesar de estar convencidos de la base neurológica de estas perturbaciones, la neurología no cuenta con elementos específicos para su terapéutica (Orlievsky, 1999).

La neuropediatra Rappin (citada por Orlievsky, 1999), "señala que en relación con la etiología del autismo, es importante indicar que todavía su diagnóstico continúa siendo completamente clínico, ya que en la actualidad no existe ningún examen biológico que pueda validarlo a través de la demostración de una disfunción del sistema nervioso. Para la autora, el autismo puede tener una etiología genética o puede ser una consecuencia de la rubéola congénita, espasmos infantiles, esclerosis tuberosa u otras causas, en los cuales aparecen algunos casos desencadenados por situaciones traumáticas de origen emocional" (Orlievsky, 1999).

"De un modo u otro, sea la etiología de origen genético, orgánico o emocional, el diagnóstico se hace con base en las manifestaciones de la conducta... A esta oposición entre orgánico y emocional se suma otra cuestión no menos importante; si en estos casos se trata de una psicosis infantil consecuencia de una falla en la estructura, contrapuesto al de la psiquiatría que abandona la noción de psicosis en la infancia creando en su lugar la noción de trastorno generalizado del desarrollo. Este criterio da cuenta de trastornos en la adquisición de variadas funciones psicológicas fundamentales, relativas a la adquisición de aptitudes sociales y del lenguaje" (Orlievsky, 1999). Chinchilla,
(2001) considera que Aserment (1997) sugiere una respuesta concisa ante las múltiples teorías organicistas que se han adjudicado el descubrimiento de la verdad en la etiología del autismo, optando por una visión reduccionista de la complejidad del fenómeno.

\section{METODOLOGÍAS Y PROCEDIMIENTOS}

El diseño más empleado en las investigaciones costarricenses revisadas es el tipo: sujeto único (Calderón y Chacón, 2000), porque permite un adecuado acercamiento a la población y a las características en estudio (Soto, 1994; Cabezas, 1989). Mayor y Labrador (1984) consideran que estudios cuidadosos de casos aislados, pueden revelar factores de importancia fundamental acerca del fenómeno estudiado $y$ han demostrado ser sumamente eficaces en la práctica de modificación de conducta y la psicología clínica aplicada (Márquez y Pérez, 1994).

Dentro de este se ha utilizado, por los mismos autores mencionados, una conjugación propuesta por el investigador Fonseca (1989, citado por Soto, 1994), denominada diseño de Múltiples $\mathrm{AB}$ que se trabaja en dos fases: el establecimiento de la línea base y la etapa de intervención. Básicamente las ventajas destacadas de este subdiseño, es que permite obtener un análisis intersujeto e intrasujeto, dando mayor solidez a los resultados; también posibilita participar en cualquier momento ya que no hay interdependencia entre la línea base y la intervención de cada sujeto (Fonseca, 1987).

Para la obtención y análisis de los datos se han diseñado instrumentos para el registro de eventos, para la observación de frecuencias, conteo de frecuencias, registro de habla signada, registro de línea base e intervención, reforzadores, y el registro anecdótico. También se emplean técnicas estadísticas descriptivas (Calderón, y Chacón 2000; Chacón, y otros, 1994).

La mayoría de estudios son de tipo exploratorio, con metodologías cuantitativas y cualitativas dirigidos a la investigación acerca de las conductas que interfieren en el aprendizaje $y$ las conductas sociales, pero desde el punto de vista de padres y maestros. 


\section{CONCLUSIONES}

El estudio del autismo ha avanzado a grandes pasos, a los niveles cualitativo y cuantitativo, la cantidad de estudios realizados $y$ temas abordados en la investigación son prueba de ello. Aunque todavía quedan muchas dudas $y$ aspectos por descubrir y aclarar, es claro que el desarrollo de esta área temática es de gran importancia en la actualidad. Todavía queda por definir con mayor claridad las conductas asociadas y exclusivas del trastorno; este logro incidirá con gran peso en el establecimiento del diagnóstico diferencial $y$ dará pie al desarrollo de mejores y eficaces técnicas.

En un principio las dificultades del diagnóstico diferencial eran abundantes, por la similitud sintomática y la proximidad etiopatogénica con otros cuadros al ser un trastorno del desarrollo. Aunque no se ha encontrado la etiología ni el tratamiento específico, sí se han realizado importantes descubrimientos respecto a cuáles son más eficaces y cuáles utilizar para síntomas y conductas específicas. Se ha descubierto también que aunque no es una enfermedad reversible porque conlleva un daño en el desarrollo, puede ser minimizado mediante diversas técnicas de educación especial que se apoyan interdisciplinariamente en la psicología, psiquiatría, medicina, biología, pediatría, trabajo social, etc.

En este sentido, las investigaciones costarricenses y extranjeras apuntan a la necesidad de una fusión entre la terapia y la educación que integre al medio y a las personas significativas para el niño. Si se quieren lograr mejores resultados, en este campo como en tantos otros, el trabajo interdisciplinario es un elemento indispensable.

La integración de los métodos psicológicos y pedagógicos es ya un gran paso, pues es fundamental si se quiere avanzar en la intervención terapéutica, apuntando a una mejor socialización y desarrollo general del niño. El estado actual muestra grandes controversias pero también es reflejo del esfuerzo de integración desde diversas disciplinas.

\section{REFERENCIAS BIBLIOGRÁFICAS}

\section{INVESTIGACIONES}

Cabezas P. H. "El uso de técnicas de modificación de conducta para la adquisición de destrezas básicas y pre-académicas en cinco niños autistas costarricenses". Tesis (Magister Scientiae)Universidad de Costa Rica. Comisión del Programa de Estudios de Posgrado en Rehabilitación Integral, San José, Costa Rica. 1989.

Calderón, C. E. y Chacón, C. M. "Desarrollo de destrezas comunicativas funcionales en niños que presentan el síndrome de autismo: aplicación del programa de habla signada de Schaeffer y colaboradores (1982)". Memoria de Práctica Dirigida para optar por el grado de Licenciatura en Ciencias de la Educación Especial con énfasis en Retos Múltiples. Universidad de Costa Rica, Facultad de Educación, Escuela de Orientación y Educación Especial. 2000.

Chacón, V. D.; Vargas, D. K.; Padilla, L. M.; Cayasso, O. G.; Muñoz, S. I. y Hernández, V. E. "Entrenamiento inicial en comunicación funcional por medio de una tabla de comunicación para niños y adolescentes con discapacidad múltiple y severa, utilizando el "time delay" y el Sistema de procedimientos de instigación gradual”. Memoria del Seminario de Graduación para optar por el grado de Licenciatura en Educación Especial con énfasis en discapacidad múltiple y severa. Universidad de Costa Rica, Facultad de Educación, Escuela de Orientación y Educación Especial. 1994.

Chinchilla, I. “¿Debe el psicoanálisis hacer una relectura de sus teorías acerca del síndrome autista, ante los descubrimientos de otras disciplinas en dicha materia?". Trabajo realizado para el curso Investigación VI, I semestre 2001. 
Fernández, P. K.; Morales, B. A.; y Sauma, F. R. "Una estrategia de enseñanza de la lectura para personas con el síndrome de autismo". Memoria de Práctica Dirigida para optar por el grado de Licenciatura en Ciencias de la Educación Especial con énfasis en Retos Múltiples. Universidad de Costa Rica, Facultad de Educación, Escuela de Orientación y Educación Especial. 1999.

Márquez, C. M. y Pérez G. D. "Entrenamiento a padres de niños con autismo, de la Escuela Neuropsiquiátrica Infantil, en el uso de técnicas de modificación de conducta, utilizando el "Taller de Modificación de Conducta abcs para padres de Rettig": una alternativa co-terapéutica". Tesis para optar por la Licenciatura en Psicología, Universidad de Costa Rica. Facultad de Ciencias Sociales. Escuela de Psicología, San José, C. R. 1994.

Rodríguez, R. V. "Análisis comparativo de los puntajes promedio obtenidos por niños autistas estadounidenses y costarricenses en la lista de cotejo para la conducta autista". Tesis de Licenciatura en Ciencias de la Educación con énfasis en incapacidad múltiple. Universidad de Costa Rica. Facultad de Educación, San José, C. R. 1992.

Soto, C. R. "Un sistema alternativo en la enseñanza del lenguaje a niños con autismo: comunicación total". Tesis de Licenciatura en Educación Primaria, Universidad de Costa Rica. Facultad de Educación. Escuela de Formación Docente, San José, C. R. 1994.

REVISTAS

Aserment, F. "La cuestión del sujeto en el tratamiento psicoanalítico del autismo". En: Revista de la Asociación Española de Neuropsiquiatría, 1997, nro. 63.
Baron-Cohen y otros. "El déficit de la Teoría de la Mente en autismo: algunas cuestiones para la enseñanza y el diagnóstico": CHAT Cuestionario para detección de riesgo de Autismo a los 18 meses. En: Revista Siglo Cero, Nov-Dic. España. 1993.

Creak M., Ini S. "Families of psychotic children”. En: Journal Children Psychology, 1960, nro. 1, págs.156-175.

Delgado, Celia. "Psicoanálisis y psiquiatría: ¿abordajes compatibles en su perspectiva del autismo?". En: Revista Psicología Iberoamericana, 1996. Vol. 4, págs. 11-23.

Frith, Uta. "El autismo". En: Revista Investigación y Ciencia, 203, 1993, págs. 58-65.

Fonseca, R. G. "Un programa de tratamiento para el autismo". En: Educación(C. R.). Vol. 11, nro. 1, 1987, págs. 77-80.

Guzmán, W. (1995). "Nuevos conocimientos sobre autismo infantil". En: Revista de Educación. (Chile). 1995. Nro. 230.

Pérez de Plá, E. "Autismo y psicoanálisis". En: Revista Psicología Iberoamericana, Vol. 4. 1996. págs. 29-49.

Sánchez, G. "El vínculo emocional en el autismo". En: Revista Psicología Iberoamericana. Vol. 4, 1996, págs. 5-9.

Trejo, G. "Detrás de una muralla de silencio". En: Revista Psicología Iberoamericana, Vol. 4, 1996, págs. 50-53.

\section{ARTÍCULOS EN INTERNET}

Autism Society of America: <www.autismsociety.org>

Hernández, L. "El psicoanálisis, eje para el tratamiento del autismo". En: Vida 
Universitaria. Semanario de la Universidad Autónoma de México. 1997. Vol IV, nro.14. México, DF. Diciembre. <http://www.uam.mx/organo-uam/ documentos/V-IV/iv14-14.html>

National Institute of Mental Health. <www. nimh.nih.gov>

National Institute of Child Health. <www.nichd. nih.gov>

(NAAR). En: <http://www.naar.org>

Orlievsky, D. "Perspectivas clínicas en el abordaje del autismo y otros trastornos profundos del desarrollo". Seminario Psicoanálisis con niños. Nro. 20. 1999. www.edupsi.com/psa-ninos.htm

Rimland B., (1964) The Autism Explosion. En: Autism Research Institute: $<w w w$. autism-institute.com>

Revista Autismo en sociedad. Año II, nro. 9 Agosto 1997 AMs Producciones. En: $<$ http://www.autism.org/temple/inside. html>

OTROS

American Psychiatric Association. DSM-IV. Manual diagnóstico y estadístico de los trastornos mentales. $1^{\text {era }}$ de. Masson. 1995.

Baron-Cohen, S. Mind-blindness. An essay on Autism and Theory of mind. United States of America. The Mit Press. 1999.

Bender, L y Maslow, A. Trastornos mentales y emocionales. 1. ed. Buenos Aires, Arg. Paidós. 1965.

Bettelheim, B. La fortaleza vacía: el autismo infantil y el nacimiento del sí mismo. 2. Ed. Barcelona: Laia. 1977.
Bleuler, E. Psiquiatria. Rio de Janeiro: Edit. Guanabara. 1985.

Brauner, A y Brauner, F. Vivir con un niño autístico. Barcelona, Ed. Paidós. 1978.

Kanner, L. A word to parents about mental hygiene. Madison: University of Wisconsin Press. 1957.

Klein, M. El psicoanálisis de niños. Buenos Aires: Asociación Psicoanalítica Argentina. 1948.

Lien de Rozental, M.E. El autismo, enfoque fonoaudiológico: el síndrome del autismo infantil con especial referencia a los problemas perceptuales de comunicación, habla y lenguaje. Buenos Aires, Arg. Edit. Médica Panamericana, c1983.

Mahler, M. EI nacimiento psicológico del infante humano. Buenos Aires. Ed. Marymar. 1975.

Mayor, J. y Labrador F. J. Manual de modificación de conducta. Madrid. Ed. Alhambra. 1984.

Negrón, R. "Hallazgos clínicos y bioquímicos en una muestra de 100 niños autistas". Ponencia presentada en International Symposium on Autism Research, Boston, Mass, Julio 14-18. 1981.

Paluszny, M. Autismo: Guía práctica para padres y profesionales. México. Ed. Trillas. 1987.

Rutter M., y Schopler E. Autismo: reevaluación de los conceptos y el tratamiento. Versión española A. López-Lago, Ed. Madrid. Alhambra. 1984. 
Tustin, F. Autismo y psicosis infantiles. Barcelona. Ediciones Paidós. 1972.

Wing, L. La educación del niño autista: guía para los padres y maestros. Barcelona. Piados. 1981.

\section{BIBLIOGRAFÍA GENERAL}

Baron-Cohen, S. "Social and pragmatic deficits in autism". Journal of the Autism and Developmental Disorders. Nro. 18, 1976: 379-402.

Churchill, D. W y DeMyer, M. K. "The relation of infantile autism and childhood schizophrenia to developmental language disorders of childhood". Journal of autism of childhood schizophrenia. Vol.2, 1971: 182-197.

Coleman, M y Gillberg, Ch. El autismo: Bases biológicas. Editorial Martínez Roca. S.A. Barcelona, España. 1989.

Diez Cuervo, A y Martos, J. "Definición y etiología”. En: Intervención Educativa en Autismo Infantil I. CNREE, Madrid: MEC. 1989.

DeMyer, M.K et al. Social adaptative behaviors of autistic children measuredin a structural psychiatric interview. Churchill (ed): Autism Proceedings of the Indiana University. Indiana; Springfield, III. 1968.

Eisenbert, L., Kanner, L. y Schopler. "Early infantile autism”. American Journal of Orthopsyachiatry. 26, 1956: 556-566.

Fonseca, R.G. (1983) "Repetición de la investigación. Un procedimiento para demos- trar la validez y la efectividad de los procedimientos de intervención". En: Revista Educación (U.C.R.). Vol. 3, nro. 1 y 2, 1989: pp. 37-38.

Freeman, B. J et al. "Establishing the diagnosis of autism Methodological problems". Journal of Autism and developmental disorders. Vol 14, Nro. 2 , 1981: 2-23.

- "Behavior assessement of the Syndrome of Autism: Behavior Observation System". Journal of the American Academy of Child Psychiatry. 1984.

Freeman, B. J y Ritvo E. et al. "El síndrome del autismo: estableciendo el diagnóstico y los principios de manejo". Pediatric Annal. Vol 13, Nro. 4, 1984: 284-296.

Hobson, P. "The autistic child's appraisal of expressions of emotions". Journal of Child Psychology and Psychiatric. 1993.

Lotter, V. "Epidemiology of autistic conditions in young children: I prevalence". Social Psychiatric. 1966: 124.

Polaino, A. Introducción al estudio científico del autismo infantil. Editorial Alhambra, España. 1982.

Rivière, A. "Interacción y símbolo en autistas". Infancia y Aprendizaje. 1983.

Rutter, M. Infantile Autism: Concepts, characteristics and treatment. Londres. 1971. 